\title{
Genetic and Environmental Variation in Anthocyanins and their Relationship to Antioxidant Activity in Blackberry and Hybridberry Cultivars
}

\author{
Ann Marie Connor \\ HortResearch-Ruakura Research Centre, Private Bag 3123, Hamilton, New Zealand
}

Chad E. Finn

U.S.Department of Agriculture-Agricultural Research Service, Horticultural Crops Research Laboratory, 3420 NW Orchard Avenue, Corvallis, OR 97330

\author{
Tony K. McGhie \\ HortResearch-Batchelar Research Centre, Tennent Drive, Palmerston North, New Zealand
}

Peter A. Alspach

HortResearch-Nelson Research Centre, P.O. Box 220, Motueka, New Zealand

AdDitional IndEX wORDs. phenolic, Rubus, phenotypic correlation

\begin{abstract}
Dietary anthocyanins (ACYs) may give health benefits through their antioxidant activity (AA) or other physiological effects. We examined AA and ACY profiles and contents in 16 blackberry and hybridberry (Rubus L. species) cultivars harvested in 2002 and 2003 in New Zealand and Oregon. Total ACY content varied widely among cultivars harvested from a single site in a single year (e.g., from 58 to $343 \mathrm{mg} / 100 \mathrm{~g}$ fruit Oregon in 2003). For the 12 cultivars common to both sites and years, cultivar and year within location significantly affected total ACY content, accounting for $40 \%$ and $10 \%$ of total variation, respectively. Cultivar interactions with both location and year within location were also significant, together accounting for $39 \%$ of variation. Cyanidin 3-O-glucoside and cyanidin 3-O-rutinoside were identified in all cultivars in both locations in at least 1 year. Compared with total ACY, cultivar accounted for more variation in these two ACYs (63\% and 92\%, respectively), while cultivar interactions together accounted for a smaller, but statistically significant, proportion of variation $(23 \%$ and $7 \%$, respectively). Cyanidin $3-O$-sophoroside and cyanidin 3-O-(2G-glucosylrutinoside) were identified in only four cultivars. Cultivar effects accounted for $64 \%$ and $76 \%$ of variation in these ACYs, respectively, while cultivar interactions together contributed $18 \%$ and $24 \%$, respectively. For AA, cultivar effects were not significant, contributing $11 \%$ of variation; in contrast, year effect and cultivar $x$ environment interactions were significant, contributing $22 \%$ and $55 \%$ of total variation, respectively. Based on cultivar means for all 16 genotypes, the phenotypic correlation between AA and total ACY was positive but lower than that between AA and total phenolic content (TPH) $(r=0.63$ and 0.97 , respectively). Combinations of individual ACYs did not provide higher correlations with AA. Thus, ACY profiles and content are not as useful as TPH as a proxy measurement for AA.
\end{abstract}

Oxidative stress of cellular lipids, proteins, and nucleic acids has been implicated in the development of several chronic and debilitating human diseases that occur frequently in industrialized nations, including atherosclerotic cardiovascular disease, cancer, neurodegenerative diseases (e.g., Parkinson's and Alzheimer's diseases), and others (Cadet and Brannock, 1998; Esterbauer et al., 1992; Leake, 1998; Markesbury, 1997; Praticò, 2002). Cellular oxidation is usually induced by free radical species produced by normal cellular processes or encountered in our environment. The body's endogenous antioxidant enzymes work to prevent cellular oxidation, but dietary antioxidant micronutrients, such as vitamin $\mathrm{E}$, also play central roles in controlling oxidative stress. Other dietary compounds with antioxidant activity (AA), such as the simple and complex phenolics that are present in foods of plant but not animal origin, may serve similar functions. These phenolic antioxidants might partly explain the inverse association observed in some epidemiological studies between fruit and vegetable consumption and incidence of, or mortality from, cardiovascular

Received for publication 3 Feb. 2005. Accepted for publication 3 Apr. 2005. We thank Mary Peterson and Laura Barnett for technical assistance; Nnadozie Oraguzie and Ron Beatson for reviewing an earlier version of the manuscript; and Harvey Hall for access to the blackberry and hybridberry germplasm collection at HortResearch. disease and cancer (Block et al., 1992; Hertog et al., 1997; Knekt et al., 2002; Steinmetz and Potter, 1996). Included among the complex phenolics are the flavonoids, of which anthocyanins (ACYs) are one class. In vitro AA of individual ACYs or of ACY-enriched fruit extract fractions has been demonstrated in several assay systems (Deighton et al., 2002; Mullen et al., 2002; Stintzing et al., 2002; Tsuda et al., 1994; Wang et al., 1997). In general, their AAs appear to compare favourably with those of other flavonoid classes (Fukumoto and Mazza, 2000), and some reports of in vitro and in vivo animal studies propose that the AA is important to the potential health benefits of ACYs, although other activities, such as anti-inflammatory activity, might contribute to their health benefits (Andriambeloson et al., 1998; Rossi et al., 2003; Serraino et al., 2003; Youdim et al., 2000). However, the contribution that $\mathrm{ACY}$ s make to AA in fruit is less clear; the concentrations and relative AA of the other phenolic antioxidants (e.g., flavonols, phenolic acids, flavanols) and nonphenolic antioxidants (e.g., vitamin $\mathrm{C}$ ) present in the fruit will alter the significance of the contribution made by ACYs.

Among fruits, berry fruits in particular are rich in ACYs and the contribution that ACYs make to AA will be of interest to breeders whose goals include improving fruit color quality and AA to produce more healthful fruit. The relationship between 
ACY and AA in Rubus fruits, and specifically blackberries and hybridberries, has been addressed in a few studies. [For clarity we use "blackberry" to refer to genotypes whose known or presumed ancestry includes only representatives of the Rubus blackberry subgenera, and "hybridberry" to refer to those genotypes whose fruit pick as blackberries (i.e., the receptacle remains with the fruit) but whose ancestries also include nonblackberry species, such as $R$. idaeus L. The distinction between these two groups is preferred by some horticulturists and breeders; however, because blackberries and hybridberries are frequently cultivated together commercially and are often cross-hybridized in breeding programs, our analyses examined them collectively, without specific intention of comparing blackberries and hybridberries.] Deighton et al. (2000) observed a phenotypic correlation of 0.37 between ACY content and the Trolox equivalent antioxidant capacity (TEAC) among representatives of 12 Rubus species tested in one year; the correlation coefficient between ACY and the ferric-reducing antioxidant power (FRAP) in these same samples was 0.59. Only some of the species represented in the study belong to the blackberry subgenera. Moyer et al. (2002) reported a correlation of 0.90 between ACY and oxygen radical absorbance capacity (ORAC) among 37 Rubus species and cultivars tested in 1 year, but a correlation of 0.70 between ACY and ORAC when the sample was limited to 27 hybrid blackberries. The correlations between ACY and FRAP for these two groups of Rubus accessions were 0.85 and 0.38 , respectively. Clark et al. (2002) reported a correlation of 0.62 between ORAC and ACY in 20 blackberry genotypes from the Univ. of Arkansas breeding program, eight of which were assayed in both years of a 2-year study. Thus, there is some inconsistency among the reported correlations, possibly due to differences in the species or environments, as well as the different AA assays employed, among the studies.

In cultivated blackberry, the major ACYs are cyanidin 3-Oglucoside (cy-glu) and cyanidin 3-O-rutinoside (cy-rut) (Sapers, et al., 1986; Siriwoharn et al., 2004; Torre and Barritt, 1977). The production of the latter ACY requires a rhamnose substitution, presumably controlled by major gene $R$, which is present in some raspberry genotypes (Barritt and Torre, 1975), and in virtually all blackberry genotypes (Jennings and Carmichael, 1980). Some species included in the blackberry subgenera of Rubus are reported to contain cyanidin-based ACYs with other sugar substitutions or with acyl substitutions (Mazza and Miniata, 1993; Stintzing et al., 2002) and low concentrations of pelargonidin-based ACYs (Deighton et al., 2000; Torre and Barritt, 1977). Additionally, hybridberries may contain ACYs usually associated with nonblackberry subgenera of Rubus (Harborne and Hall, 1964; Jennings and Carmichael, 1980).

With the exception of the report by Clark et al. (2002), which contains data from 2 years, the studies cited do not examine the effect of year or location on variation in ACY and AA. Our primary objective in this study was to determine the effects of environment (year and location) on variation in individual and total ACYs among 16 (3 blackberry and 13 hybridberry) cultivars harvested in two consecutive seasons in New Zealand and Oregon. A secondary objective was to examine the relationship between ACY and AA.

\section{Materials and Methods}

Full details of cultural practice, fruit sampling, extraction, and AA assay are provided in Connor et al. (2005a). Brief summaries of the methods are given here.
Plants And fruit. Single plots (one to three plants) of each cultivar were harvested at Nelson, New Zealand (NZ), and at Aurora or Corvallis, Ore. (OR), over two seasons (2002-03, designated 2002; and 2003-04, designated 2003). Twelve of the 16 chosen cultivars were successfully harvested in both years at both locations, with $\approx 100 \mathrm{~g}$ of shiny, sound, ripe fruit being collected when $\approx 50 \%$ of the berries on the plant were fully ripe. Fruit were frozen within 3 h of harvest. Frozen fruit from both locations were shipped on dry ice to the laboratory in Palmerston North, New Zealand, where they were held at $-80{ }^{\circ} \mathrm{C}$ until extraction.

Extraction. Duplicate extractions were conducted under reduced light conditions or under safety-light in a fume hood. Approximately $30 \mathrm{~g}$ of frozen fruit (average of four to five fruit) were weighed and homogenized with $150 \mathrm{~mL}$ solvent ( 80 ethanol : 20 water : 1 glacial acetic acid, by volume), and stored for 48 $\mathrm{h}$ at $4{ }^{\circ} \mathrm{C}$. Seeds were not separated from pulp during extraction. Following storage, the homogenate was allowed to equilibrate toward room temperature $\left(\approx 18^{\circ} \mathrm{C}\right)$ for $1 \mathrm{~h}$, before two $10-\mathrm{mL}$ aliquots were removed and centrifuged at $1500 \mathrm{~g}_{\mathrm{n}}$ for $20 \mathrm{~min}$. Approximately $4 \mathrm{~mL}$ of supernatant were removed from each aliquot and stored at $-20{ }^{\circ} \mathrm{C}$ in separate amber glass vials until assayed. The same extracts were used for all assays.

AnTHOCYANIN ANALYSES. Individual ACYs were determined in the extracts by HPLC (Connor et al., 2005b). Values are reported for four individual ACYs (cy-glu, cy-rut, cy-sop, and cy-glurut) that together comprised $>90 \%$ of total ACYs in most of the cultivars and eluted between 7 and 15 min after the solvent program started. Additionally, ACY peaks with long retention times (LRPs), eluting at 15 to $30 \mathrm{~min}$, were collectively quantified. Total ACYs included all ACYs that eluted between 7 and $30 \mathrm{~min}$. Cyanidin 3-O-galactoside, cyanidin 3-O-glucoside, and cyanidin 3-Oarabinoside (Polyphenols Laboratories, Sandes, Norway) were used as standards. The ACY components were quantified using an external calibration and expressed as cyanidin 3-O-galactoside equivalents in $\mathrm{mg} / 100 \mathrm{~g}$ fruit. Components were identified by comparison with the ACY profile of 'Boysen', which had been previously determined (Cooney et al., 2004).

Antioxidant ACTIVITy. The ferric-reducing antioxidant power (FRAP, also referred to as the "ferric-reducing activity of plasma") (Benzie and Strain, 1996) assay was used, with modifications by Deighton et al. (2000). Results are expressed as ferrous sulfate equivalents (FE) in $\mu \mathrm{mol} \cdot \mathrm{g}^{-1}$ fruit.

Statistical ANALYSEs. The analyses of variance and variance component derivations are described in Connor et al. (2005a). All factors, including cultivars, were treated as random effects. Phenotypic correlations were calculated using cultivar means over years and locations. In addition, linear modeling for AA was performed using stepwise elimination, based on Akaike's information criterion, of terms from a full model, which incorporated individual ACYs and their first-order interactions (Venables and Ripley, 1994). Various clustering methods, including linear discriminant function analysis and classification trees, were explored to investigate whether partitioning of the cultivars based on the ACY profile corresponded with their lineages. All analyses were performed using S-Plus software (version 6.1.2, release 1 for Microsoft Windows 2002; Insightful Corp., Seattle, Wash.).

\section{Results}

Among the 12 blackberry and hybridberry cultivars harvested in both locations in both years, the range in total ACY content was similar in NZ (75.9-164.1 mg/100 g fruit) and in OR (80.4-183.5 
$\mathrm{mg} / 100 \mathrm{~g}$ fruit) in 2002, and slightly less in NZ (66.0-167.8 $\mathrm{mg} / 100 \mathrm{~g}$ fruit) than in OR $(58.3-219.3 \mathrm{mg} / 100 \mathrm{~g}$ fruit) in 2003 (Table 1). Although for many cultivars, mean values within a location were similar in 2002 and 2003, there were some that showed considerable within-location between-year differences. For cy-glu, which was detected in all of the cultivars and represented the major ACY in most of them, the range in values was wider in OR than in NZ in both years, while for cy-rut the ranges were wider in NZ than in OR in both years. Cy-rut was present at lower concentrations than cy-glu in all cultivars, but demonstrated a wide range of means, and it was not present in all location-year combinations. For example, it was detected in 'Hull Thornless' in 2002 in both locations but was not detected in fruit harvested in both locations in 2003. Cy-sop and cy-glurut were detected in both years and both locations only in 'Boysen', 'Logan', 'Ranui', and 'Tayberry' (Table 2). They were not identified in any of the other cultivars, except 'Silvan' and 'Siskiyou' (cy-sop only) in a single year and location (data not shown). In NZ, cy-sop content of 'Boysen' and 'Logan' approximated or exceeded cy-glu content in both years. The ACY peaks characterized as LRPs were not specifically identified for this study, although some may represent acylated anthocyanins. The LRPs displayed a range of values among cultivars that was similar in each of the 4-year location combinations ( $\approx 1-20 \mathrm{mg} / 100 \mathrm{~g}$ fruit), and were minor constituents in most cultivars. However, in a few cultivars (e.g., 'Hull Thornless' and 'Navaho') the LRPs accounted for a substantial proportion of the total ACYs in each environment. Two representative chromatograms are shown in Fig. 1.

The ranges in AA values were similar in the two locations in 2002: from 49.0 to $81.0 \mu \mathrm{mol} \cdot \mathrm{g}^{-1}$ fruit in $\mathrm{NZ}$ and from 58.7 to $83.0 \mu \mathrm{mol} \cdot \mathrm{g}^{-1}$ fruit in OR. In 2003 , the range was narrower in NZ than in OR. In some instances, the within-cultivar between-year changes in AA were quite different from those that occurred in total or individual ACYs. For example, the substantial changes in total ACY and cy-glu observed in 'Black Butte' between years in both locations was accompanied by only modest changes in AA between years.

The four additional cultivars that were not harvested in both years in both locations contained total ACY, cy-glu, and cy-rut in concentrations that were consistent with those of the other 12 cultivars, with the exception of 'Chehalem'. The values for total ACY and cy-glu for this cultivar were substantially higher than those of the remaining cultivars (Table 1).

The importance of cultivar to total variation in ACY content is demonstrated by the variance component distribution analysis (Table 3). The cultivar main effect accounted for only $40 \%$ of total variation in total ACY, but its interactions accounted for an additional $38 \%$. For all individual ACYs and LRPs, the main effect of cultivar was significant, or nearly so, and accounted for $64 \%$ to $93 \%$ of total variation. Cultivar interactions with location and/or year within location accounted for further variation, particularly for those ACYs for which the cultivar main effect contributed $<90 \%$. In contrast, location and year main effects accounted for $<5 \%$ and $\leq 7 \%$ of variation, respectively, and rarely achieved statistical significance. The location effect contributed little to variation in total ACY, whereas the year within location effect was significant and contributed $10 \%$ to total variation.

The distribution of variance components for AA differed substantially from those for the ACYs; cultivar main effect accounted for only $11 \%$ of variation and was not significant, while year effects account for $22 \%$. Additionally, the cultivar interactions account for $55 \%$ of total variation.
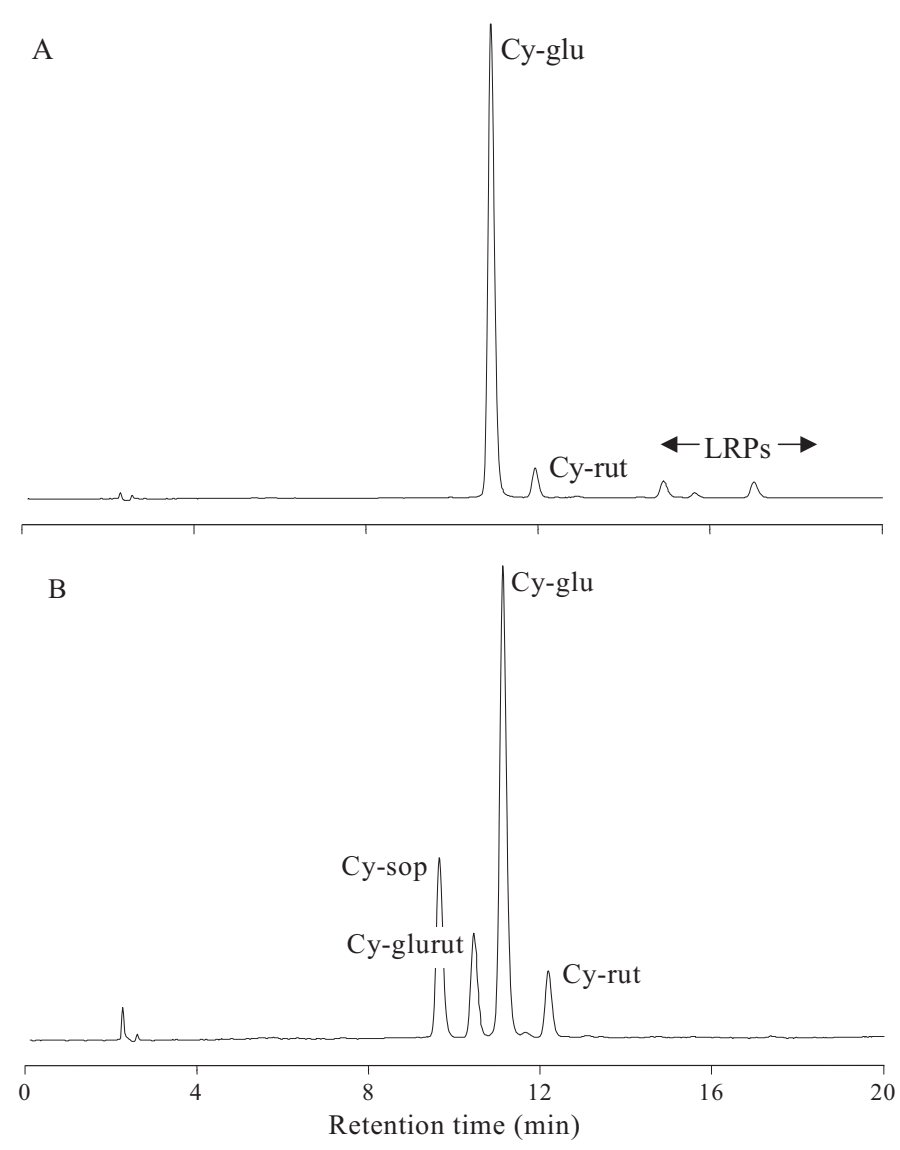

Fig. 1.Two representative HPLC chromatogram traces showing the anthocyanin peaks for (A) 'Chehalem' in New Zealand in 2002, and (B) 'Boysen' in Oregon in 2003. The retention times of the individual anthocyanins vary slightly between these two traces because they were selected from different years. This variation is within normal operating limits for HPLC and peak identity was verified by authentic anthocyanin standards. Abbreviations for anthocyanins: Cy-glu $=$ cyanidin 3-O-glucoside, Cy-rut $=$ cyanidin 3-O-rutinoside, $\mathrm{Cy}$-sop $=$ cyanidin 3-O-sophoroside, $\mathrm{Cy}$-glurut $=$ cyanidin $3-O-\left(2^{\mathrm{G}}\right.$-glucosylrutinoside $)$, and LRPs $=$ anthocyanin peaks with retention times longer than $15 \mathrm{~min}$.

For all analyses, variation among samples was statistically significant. However, it accounted for only $7 \%$ to $8 \%$ of the variance in total $\mathrm{ACY}$ and $\mathrm{AA}$, and accounted for $<5 \%$ for the individual anthocyanins and LRPs.

Because an inverse correlation between fruit weight and some ACYs, and between fruit weight and AA, was noted (Table 4), analyses were repeated using fruit weight as a covariate. For ACYs, the significance of the main effects and interactions were not substantially altered with adjustment for weight (analyses not shown). However, for AA, adjustment for fruit weight altered the analysis, such that cultivar accounted for $29 \%$ of the total variance and approached significance $(P=0.057)$ (Connor et al., 2005a).

Among all cultivars tested, the correlation between total ACY and cy-glu was high $(r=0.90)$ and between total ACY and cy-rut was low $(r=0.22)$, reflecting the role of cy-glu as the major contributor to total ACY in most cultivars (Table 4, top). The correlation between cy-glu and cy-rut was negligible $(r=$ 0.06). The correlations between total ACY, cy-glu, or cy-rut and cy-sop or cy-glurut were all low to moderate and negative when all cultivars were included. Since only four cultivars produced cy-sop and cy-glurut on a consistent basis, correlations obtained by considering only these four were also obtained (Table 4, bot- 
Table 1. Mean values of total anthocyanins (ACY), cyanidin 3-O-glucoside, cyanidin 3-O-rutinoside, and antioxidant activity (by FRAP assay) in fruit from 16 blackberry and hybridberry cultivars harvested in 2002 and 2003 in Oregon (OR) and New Zealand (NZ). Cultivars are listed in increasing order of mean total ACY over all environments.

\begin{tabular}{|c|c|c|c|c|c|c|c|c|c|c|c|c|c|c|c|c|}
\hline \multirow[b]{3}{*}{ Cultivar } & \multirow{2}{*}{\multicolumn{4}{|c|}{\begin{tabular}{l}
\multicolumn{2}{c}{ Total ACY } \\
(mg/100 g fruit) \\
NZ $\quad$ OR
\end{tabular}}} & \multirow{2}{*}{\multicolumn{4}{|c|}{$\begin{array}{c}\text { Cyanidin } 3-O \text {-glucoside } \\
\text { (mg/100 g fruit) } \\
\mathrm{NZ} \quad \text { OR }\end{array}$}} & \multicolumn{4}{|c|}{$\begin{array}{c}\text { Cyanidin 3-O-rutinoside } \\
\text { (mg/100 g fruit) }\end{array}$} & \multicolumn{4}{|c|}{$\begin{array}{c}\text { Antioxidant activity } \\
\left(\mu \mathrm{mol} \cdot \mathrm{g}^{-1} \text { fruit }\right)\end{array}$} \\
\hline & & & & & & & & & \multicolumn{2}{|c|}{$\mathrm{NZ}$} & \multicolumn{2}{|c|}{$\mathrm{OR}$} & \multicolumn{2}{|c|}{ NZ } & \multicolumn{2}{|c|}{ OR } \\
\hline & 2002 & 2003 & $\frac{\text { OR }}{2002 \quad 2003}$ & 2003 & \multicolumn{2}{|c|}{$\frac{\text { NL }}{2002 \quad 2003}$} & 2002 & 2003 & 2002 & 2003 & 2002 & 2003 & 2002 & 2003 & 2002 & 2003 \\
\hline Logan & 75.9 & 66.0 & 80.4 & 101.8 & 22.5 & 23.2 & 40.0 & 39.5 & 4.7 & 5.1 & 7.4 & 10.0 & 67.1 & 56.0 & 71.7 & 63.3 \\
\hline Tayberry & 85.4 & 74.4 & 86.5 & 92.5 & 33.7 & 33.6 & 25.5 & 17.8 & 14.3 & 15.1 & 10.7 & 9.3 & 52.1 & 40.0 & 79.1 & 58.3 \\
\hline Hull Thornless & 108.2 & 97.2 & 84.7 & 58.3 & 86.1 & 75.8 & 64.2 & 46.0 & 0.7 & 0.0 & 0.7 & 0.0 & 63.5 & 54.6 & 62.2 & 43.3 \\
\hline Shawnee & 102.6 & 154.0 & 114.8 & 128.2 & 90.9 & 140.5 & 103.6 & 116.9 & 6.0 & 7.7 & 6.1 & 7.2 & 49.0 & 56.1 & 58.7 & 51.2 \\
\hline Ranui & 111.3 & 121.4 & 104.4 & 164.8 & 63.5 & 72.4 & 68.8 & 117.8 & 0.9 & 0.5 & 1.2 & 1.4 & 68.3 & 56.1 & 70.5 & 75.1 \\
\hline Siskiyou & 119.6 & 115.3 & 130.2 & 156.4 & 78.5 & 75.1 & 95.5 & 122.9 & 38.0 & 40.2 & 32.3 & 32.8 & 74.2 & 56.2 & 71.5 & 73.3 \\
\hline Black Butte & 109.2 & 136.4 & 99.5 & 187.7 & 105.1 & 134.3 & 97.1 & 185.0 & 1.9 & 2.1 & 0.9 & 0.6 & 69.4 & 63.8 & 60.6 & 66.6 \\
\hline Silvan & 123.6 & 115.7 & 149.4 & 151.9 & 79.8 & 70.2 & 101.2 & 114.0 & 42.1 & 45.5 & 29.4 & 37.9 & 67.8 & 52.8 & 79.8 & 62.4 \\
\hline Navaho & 139.7 & 152.2 & 129.0 & 139.4 & 117.9 & 132.5 & 111.4 & 121.5 & 0.8 & 0.0 & 0.8 & 0.6 & 72.2 & 63.1 & 61.0 & 55.8 \\
\hline Boysen & 107.3 & 102.0 & 142.9 & 208.6 & 24.1 & 37.9 & 55.5 & 117.9 & 2.4 & 2.2 & 7.8 & 16.6 & 70.7 & 57.8 & 82.6 & 89.8 \\
\hline Marion & 164.1 & 167.8 & 156.4 & 171.7 & 102.8 & 112.9 & 108.1 & 130.3 & 53.0 & 48.5 & 40.8 & 35.7 & 81.0 & 70.4 & 80.8 & 70.2 \\
\hline Kotata & 133.5 & 132.4 & 183.5 & 219.3 & 121.8 & 122.2 & 168.4 & 207.8 & 9.5 & 10.2 & 12.5 & 11.5 & 60.4 & 52.2 & 83.0 & 78.4 \\
\hline $\begin{array}{l}\text { Yearly means } \\
(12 \text { cultivars })\end{array}$ & $\underbrace{115.0}$ & $\underbrace{119.6}$ & $1 \underbrace{21.8}$ & $\overbrace{}^{148.4}$ & $\underbrace{77.2}$ & 85.9 & $\underbrace{86.6}$ & 111.5 & 14.5 & 14.7 & 12.5 & 13.6 & $\underbrace{66.3}$ & 56.6 & 71.8 & 65.6 \\
\hline $\begin{array}{l}\text { Location means } \\
\text { (12 cultivars) }\end{array}$ & 11 & & & & & 31.6 & 99 & & & & 13 & & & & & \\
\hline Aurora & 101.3 & 102.3 & 131.3 & ----- & 93.6 & 93.9 & 122.9 & ----- & 7.0 & 8.4 & 7.4 & ---- & 70.2 & 54.5 & 85.2 & ---- \\
\hline ORUS 1826 & 128.8 & 135.4 & ----- & ---- & 119.4 & 129.2 & ----- & ----- & 6.7 & 6.3 & ----- & ----- & 66.5 & 56.6 & ----- & ----- \\
\hline Waldo & ----- & ----- & 142.3 & 134.7 & ----- & ----- & 128.7 & 119.9 & ----- & ---- & 9.3 & 11.5 & ----- & ----- & 79.4 & 69.0 \\
\hline Chehalem & 279.9 & ----- & ----- & 343.4 & 243.2 & ----- & ----- & 305.7 & 14.6 & ---- & ----- & 14.0 & 94.2 & ----- & ---- & 87.7 \\
\hline $\begin{array}{l}\text { Yearly means } \\
\text { (all cultivars) }\end{array}$ & $\underbrace{126.0}$ & 119.5 & $1 \underbrace{123.9}$ & $\overbrace{}^{161.3}$ & $\underbrace{92.2}$ & $\stackrel{89.6}{ }$ & $\underbrace{92.2}$ & 125.9 & $\underbrace{13.5}$ & 13.7 & 11.9 & 13.5 & $\underbrace{68.4}$ & 56.4 & 73.6 & 67.5 \\
\hline $\begin{array}{l}\text { Location means } \\
\text { (all cultivars) }\end{array}$ & & & & & & 0.9 & & & & & & & & & & \\
\hline
\end{tabular}

Table 2. Mean values of cyanidin 3-O-sophoroside, cyanidin 3-O-( $2^{\mathrm{G}}$-glucosylrutinoside), and anthocyanin peaks with retention times longer than 15 min by HPLC (LRPs) in fruit from only the blackberry and hybridberry cultivars expressing those anthocyanins ${ }^{\mathrm{z}}$, harvested in 2002 and 2003 in Oregon (OR) and New Zealand (NZ).

\begin{tabular}{|c|c|c|c|c|c|c|c|c|c|c|c|c|}
\hline \multirow[b]{3}{*}{ Cultivar } & \multicolumn{4}{|c|}{$\frac{\text { Cyanidin 3- } O \text {-sophoroside }}{(\mathrm{mg} / 100 \mathrm{~g} \text { fruit })}$} & \multicolumn{4}{|c|}{$\frac{\text { Cyanidin } 3-O-\left(2^{\mathrm{G}} \text {-glucosylrutinoside }\right)}{(\mathrm{mg} / 100 \mathrm{~g} \text { fruit })}$} & \multicolumn{4}{|c|}{$\frac{\text { LRPs }}{(\mathrm{mg} / 100 \mathrm{~g} \text { fruit) }}$} \\
\hline & \multicolumn{2}{|c|}{$\mathrm{NZ}$} & \multicolumn{2}{|c|}{ OR } & \multicolumn{2}{|c|}{$\mathrm{NZ}$} & \multicolumn{2}{|c|}{ OR } & \multicolumn{2}{|c|}{$\mathrm{NZ}$} & \multicolumn{2}{|c|}{ OR } \\
\hline & 2002 & 2003 & 2002 & 2003 & 2002 & 2003 & 2002 & 2003 & 2002 & 2003 & 2002 & 2003 \\
\hline Logan & 35.7 & 28.5 & 25.1 & 38.9 & 12.3 & 9.2 & 7.4 & 13.4 & ----- & $\begin{array}{l}---- \\
\end{array}$ & ----- & ----- \\
\hline Tayberry & 19.9 & 14.2 & 25.7 & 30.2 & 16.7 & 11.6 & 23.5 & 33.9 & ----- & ----- & ----- & ----- \\
\hline Ranui & 44.5 & 46.9 & 32.7 & 44.6 & 1.8 & 1.6 & 1.6 & 1.0 & ----- & ----- & ----- & ----- \\
\hline Boysen & 56.8 & 48.7 & 51.6 & 47.5 & 21.7 & 12.8 & 25.5 & 26.6 & ----- & ----- & ----- & ----- \\
\hline Yearly means & 39.2 & 34.6 & 33.8 & 40.3 & 13.1 & 8.8 & 14.5 & 18.7 & ----- & ----- & ----- & ----- \\
\hline Location means & \multicolumn{2}{|c|}{36.9} & \multicolumn{2}{|c|}{37.0} & \multicolumn{2}{|c|}{11.0} & \multicolumn{2}{|c|}{16.6} & & & & \\
\hline $\begin{array}{l}\text { Hull Thornless } \\
\end{array}$ & $\begin{array}{l}---- \\
\end{array}$ & $\begin{array}{l}---- \\
\end{array}$ & ----- & ----- & ----- & ----- & ----- & ----- & 21.6 & 21.4 & 19.9 & 12.3 \\
\hline Shawnee & ----- & ----- & ----- & ----- & ----- & ----- & ----- & ----- & 5.2 & 5.8 & 4.9 & 4.1 \\
\hline Black Butte & ----- & ----- & ----- & ----- & ----- & ----- & ----- & ----- & 1.3 & 0.0 & 1.1 & 2.1 \\
\hline Navaho & ----- & ----- & ----- & ----- & ----- & ----- & ----- & ----- & 20.8 & 19.7 & 16.7 & 17.3 \\
\hline Marion & ----- & ----- & ----- & ----- & ----- & ----- & ----- & ----- & 7.8 & 6.5 & 6.9 & 5.7 \\
\hline Waldo & ----- & ----- & ----- & ----- & ----- & ----- & ----- & ----- & ----- & ----- & 4.1 & 3.3 \\
\hline Chehalem & ----- & ----- & ----- & ----- & ----- & ----- & ----- & ----- & 20.9 & ----- & ----- & 23.0 \\
\hline Yearly means & ----- & ----- & ----- & ----- & ----- & ----- & ----- & ----- & 12.9 & 10.7 & 9.0 & 10.7 \\
\hline Location means & \multicolumn{2}{|c|}{----- } & \multicolumn{2}{|c|}{----- } & \multicolumn{2}{|c|}{----- } & \multicolumn{2}{|c|}{----- } & \multicolumn{2}{|c|}{11.9} & \multicolumn{2}{|c|}{9.3} \\
\hline
\end{tabular}


Table 3. Combined year proportion of total variance (PTV) and F-probabilities from the analyses of variance $(P)$ for total and individual anthocyanins ${ }^{z}$ (ACY) and antioxidant activity (AA), for 12 blackberry and hybridberry cultivars (or subsets of these cultivars $^{\mathrm{y}, \mathrm{x}}$ ) harvested in Oregon and New Zealand in both 2002 and 2003 . The numbers of degrees of freedom are shown for each group of variates, and the total variance for each variate is given in italics.

\begin{tabular}{|c|c|c|c|c|c|c|c|c|c|}
\hline \multirow[b]{2}{*}{ Source } & \multirow[b]{2}{*}{$\mathrm{df}$} & \multicolumn{2}{|c|}{ Total ACY $(n=12)$} & \multicolumn{2}{|c|}{ Cy-glu $(\mathrm{n}=12)$} & \multicolumn{2}{|c|}{ Cy-rut $(n=12)$} & \multicolumn{2}{|c|}{$\mathrm{AA}(\mathrm{n}=12)$} \\
\hline & & PTV $(\%)$ & $P$ & PTV $(\%)$ & $P$ & PTV $(\%)$ & $P$ & PTV $(\%)$ & $P$ \\
\hline Cultivar (C) & 11 & 40.3 & 0.022 & 63.5 & $<0.001$ & 92.0 & $<0.001$ & 11.1 & 0.264 \\
\hline Location (L) & 1 & 2.9 & 0.329 & 2.1 & 0.320 & 0.0 & 0.396 & 4.3 & 0.339 \\
\hline Year (Y) within L & 2 & 10.4 & 0.006 & 7.0 & 0.005 & 0.0 & 0.478 & 22.0 & $<0.001$ \\
\hline $\mathrm{C} \times \mathrm{L}$ & 11 & 19.5 & 0.022 & 10.8 & 0.033 & 5.8 & $<0.001$ & 34.6 & 0.003 \\
\hline $\mathrm{C} \times \mathrm{Y}$ within $\mathrm{L}$ & 22 & 18.6 & $<0.001$ & 12.2 & $<0.001$ & 1.3 & $<0.001$ & 20.3 & $<0.001$ \\
\hline Sample within $\mathrm{C} \times \mathrm{Y}$ within $\mathrm{L}$ & 48 & 8.1 & $<0.001$ & 4.4 & $<0.001$ & 0.8 & $<0.001$ & 7.3 & $<0.001$ \\
\hline Residuals & 95 & 0.1 & & 0.0 & & 0.0 & & 0.4 & \\
\hline Total variance & & 1471 & & 2111 & & 274 & & 138 & \\
\hline
\end{tabular}

\begin{tabular}{|c|c|c|c|c|c|}
\hline \multirow[b]{2}{*}{ Source } & \multirow[b]{2}{*}{ df } & \multicolumn{2}{|c|}{ Cy-sop $(n=4)$} & \multicolumn{2}{|c|}{ Cy-glurut ${ }^{\mathrm{y}}(\mathrm{n}=4)$} \\
\hline & & PTV $(\%)$ & $P$ & PTV $(\%)$ & $P$ \\
\hline Cultivar & 3 & 75.9 & 0.044 & 64.3 & 0.072 \\
\hline Location & 1 & 0.0 & 0.979 & 4.6 & 0.278 \\
\hline Year within L & 2 & 3.4 & 0.131 & 5.4 & 0.085 \\
\hline $\mathrm{C} \times \mathrm{L}$ & 3 & 6.9 & 0.138 & 17.6 & 0.036 \\
\hline $\mathrm{C} \times \mathrm{Y}$ within $\mathrm{L}$ & 6 & 11.4 & $<0.001$ & 7.2 & $<0.001$ \\
\hline Sample within $\mathrm{C} \times \mathrm{Y}$ within $\mathrm{L}$ & 16 & 2.2 & $<0.001$ & 0.9 & $<0.001$ \\
\hline Residuals & 32 & 0.2 & & 0.1 & \\
\hline Total variance & & 186 & & 125 & \\
\hline
\end{tabular}

\begin{tabular}{lrrr}
\hline & & \multicolumn{2}{c}{$\operatorname{LRPs}^{\mathrm{x}}(\mathrm{n}=7)$} \\
\cline { 3 - 4 } Source & df & $\operatorname{PTV}(\%)$ & \multicolumn{1}{c}{$P$} \\
\hline Cultivar & 6 & 92.6 & $<0.001$ \\
Location & 1 & 0.6 & 0.245 \\
Year within L & 2 & 0.0 & 0.496 \\
$\mathrm{C} \times$ L & 5 & 2.4 & 0.114 \\
C $\times$ Y within L & 9 & 3.7 & $<0.001$ \\
Sample within C $\times$ Y within L & 24 & 0.5 & $<0.001$ \\
Residuals & 47 & 0.1 & \\
Total variance & & 77 & \\
\hline
\end{tabular}

\footnotetext{
${ }^{\mathrm{z}}$ Abbreviations for anthocyanins: $\mathrm{Cy}$-glu $=$ cyanidin 3-O-glucoside, $\mathrm{Cy}$-rut $=$ cyanidin $3-O$-rutinoside, $\mathrm{Cy}$-sop $=$ cyanidin $3-O$-sophoroside, $\mathrm{Cy}$-glurut $=$ cyanidin $3-O-\left(2^{\mathrm{G}}\right.$-glucosylrutinoside $)$, and LRPs $=$ ACY peaks with retention times longer than 15 min in HPLC.

${ }^{y}$ Cultivars expressing Cy-sop and Cy-glurut were 'Boysen', 'Logan', 'Ranui', and 'Tayberry'.

${ }^{x}$ Cultivars expressing LRPs were 'Black Butte', 'Chehalem', 'Hull Thornless', 'Marion', 'Navaho', 'Shawnee', and 'Waldo'

(i.e., this group includes two cultivars not harvested in both locations and both years).
}

tom). The correlation between total ACY and cy-glu remained high, but that between total ACY and cy-rut was insubstantial, probably reflecting the greater contribution of cy-sop to total ACY. The correlations between total ACY and cy-sop and between cyglu and cy-sop were moderate to low but positive $(r=0.62$ and 0.41 , respectively) when only the four cy-sop-containing cultivars were included. The correlations between cy-glu and cy-glurut and between cy-rut and cy-sop in these four cultivars remained negative ( $r=-0.30$ and -0.51 , respectively), although they differed in magnitude from those based on all 16 cultivars $(r=-0.51$ and -0.27 , respectively). The correlation between cy-rut and cy-glurut was positive $(r=0.60)$ in the analysis limited to four cultivars, whereas it had been negative and low among all 16 cultivars $(r$ $=-0.13$ ). The correlations among LRPs and the other variates in the seven cultivars expressing these peaks were low.
Correlations between AA and the ACYs based on all 16 cultivars were low; the highest was that between total ACY and AA ( $r=$ $0.63)$. When the subset of four cultivars expressing cy-sop and cy-glurut were considered, the correlation coefficient was 0.66. Correlations between AA and the sums of individual ACYs were also calculated, but none matched or exceeded the correlation between total ACY and AA.

Stepwise linear modeling of AA using individual ACYs and their interactions resulted in a parsimonious model in which all individual ACYs and two interactions (cy-sop with cy-rut and cy-glurut with cy-rut) were included, with a multiple $R^{2}=0.53$ (analysis not shown). Thus, approximately half the variation in AA can be explained through ACY-based linear models. Linear discriminant function analysis, based on the cultivar ACY values for the four location $\times$ year combinations, gave a similar overall 
Table 4. Phenotypic correlations (cultivar mean basis, by year and location) among total and individual anthocyanins ${ }^{\mathrm{z}}$ (ACY), fruit weight (FW), and antioxidant activity (AA) in fruit from all blackberry and hybridberry cultivars harvested in Oregon and New Zealand in 2002 and 2003 $\left(\mathrm{n}=57\right.$, upper table); and in fruit from only those four cultivars that expressed cyanidin 3-O-sophoroside and cyanidin 3-O-( $2^{\mathrm{G}}$-glucosylrutinoside) $(\mathrm{n}=16)$ (lower table). ${ }^{\mathrm{y}}$

\begin{tabular}{|c|c|c|c|c|c|c|c|c|}
\hline & Total ACY & Cy-glu & Cy-rut & Cy-sop & Cy-glurut & LRPs & FW & AA \\
\hline Total ACY & & 0.90 & 0.22 & -0.21 & -0.21 & 0.31 & -0.31 & 0.63 \\
\hline Cy-glu & & & 0.06 & -0.51 & -0.51 & 0.38 & -0.15 & 0.45 \\
\hline Cy-rut & & & & -0.27 & -0.13 & -0.18 & 0.00 & 0.23 \\
\hline Cy-sop (all) & & & & & 0.72 & -0.38 & -0.11 & 0.10 \\
\hline Cy-glurut (all) & & & & & & -0.31 & -0.33 & 0.08 \\
\hline LRPs & & & & & & & -0.29 & 0.06 \\
\hline FW & & & & & & & & -0.44 \\
\hline Total ACY & & 0.88 & 0.06 & 0.62 & 0.13 & 0.25 & 0.17 & 0.66 \\
\hline Cy-glu & & & -0.09 & 0.41 & -0.30 & 0.19 & 0.36 & 0.50 \\
\hline Cy-rut & & & & -0.51 & 0.60 & -0.21 & -0.35 & -0.04 \\
\hline Cy-sop & & & & & 0.03 & --- & -0.25 & 0.52 \\
\hline Cy-glurut & & & & & & --- & -0.57 & 0.24 \\
\hline LRPs & & & & & & & -0.62 & 0.05 \\
\hline FW & & & & & & & & -0.25 \\
\hline
\end{tabular}

${ }^{\mathrm{z}}$ Abbreviations for individual ACYs: Cy-glu = cyanidin 3-O-glucoside, Cy-rut = cyanidin 3-O-rutinoside, Cy-sop = cyanidin 3-O-sophoroside, Cyglurut $=$ cyanidin $3-O-\left(2^{\mathrm{G}}\right.$-glucosylrutinoside $), \mathrm{LRPs}=$ ACY peaks with retention times longer than 15 min in HPLC.

${ }^{\mathrm{y}}$ In lower table, the correlations between LRPs and other variables are based on data from the seven cultivars expressing LRPs $(\mathrm{n}=28)$. Cy-sop and cy-glurut were not identified in fruit from these cultivars.

partitioning to that obtained using a classification tree approach (data not shown). However, cultivars with a high co-ancestry were not necessarily located on the same branch.

\section{Discussion}

This study demonstrates significant genotype $\times$ environment interactions for total and individual ACYs in blackberry/hybridberry cultivars. There was significant among-cultivar variation in combined year analyses for all ACYs quantified in the 12 genotypes harvested in both locations in both years. Cultivar main effects accounted for $40 \%$ to $92 \%$ of total variation in the ACYs. In contrast, variation between locations was negligible, and variation between years within location was significant but minor when compared to cultivar effects. However, both cultivar $\times$ location and cultivar $\times$ year within location interactions accounted for a considerable proportion of the variation (up to $38 \%$ together), indicating that rank or scale changes between environments occurred. Our results indicate that, since fruit color is important in fresh and processed blackberry products, assessment and selection for high total ACY in a breeding program should optimally be evaluated across several environments. Sapers et al. (1986) indicated in their study of 33 closely related thornless blackberry cultivars and selections that the genotypes could be grouped into five ACY patterns based on relative proportions of the five ACY peaks identified in HPLC analysis. They reported that the patterns were reproducible over the 2 years in which 25 of the genotypes were tested. However, while their data provide yearly mean values for each of the ACYs in each of the five patterns, they do not indicate the range of values observed within a pattern, or the ACY values in individual genotypes. Thus, their work cannot confirm the cultivar $\times$ year interaction we observed. Our study did not examine color of either the fresh or frozen fruit; thus, the association between specific ACY profiles and color was not determined. However, Sapers et al. (1986) indicated that the five ACY patterns they identified were not indicative of juice or fruit color differences observed among genotypes.

In contrast to the ACYs, variation in AA among cultivars was not statistically significant, and cultivar main effects accounted for only $11 \%$ of total variation in AA. However, year within location effect, and the cultivar $\times$ year within location and cultivar $\times$ location interactions accounted for $22 \%, 34 \%$, and $20 \%$ of total variation in AA, proportions which are nearly twice those observed in the ACY analyses. Thus, among these cultivars, the AA response to environment is less uniform than the ACY responses. The differential responses in these variables probably contribute to the low to moderate phenotypic correlations between AA and the ACYs, ranging from 0.23 for cy-rut and AA (based on all cultivars) or 0.24 for cy-glurut and AA (based on the four cultivars expressing this ACY) to 0.63 for total ACY and AA. The correlation between total ACY and AA is substantially lower than that observed between AA and TPH measured in the same fruit extracts, for which $r=0.97$ (Connor et al., 2005a). Thus, TPH would serve as a more reliable proxy measure of AA than total or individual ACYs, should direct AA assessment not be available. We also explored linear modeling for AA based on individual ACYs and their first-order interactions and found that approximately half $\left(R^{2}=0.53\right)$ the variation in AA could be explained by these factors. However, this proportion is substantially less than that explained by TPH effect $\left(R^{2}=0.93\right)$. Note that TPH, as determined by the Folin-Ciocalteu method, which is widely used and considered reliable, measures oxidizable substances, but is not entirely specific for phenolic compounds, as nonphenolic substances can interfere with the determination (Singleton et al., 1999). Thus, the true correlation between TPH and AA may be slightly lower than we report. Nevertheless, our results raise the question of whether the investment of time and reagents necessary to obtain an ACY profile by HPLC is appropriate if the principal use of the data is AA screening. As noted in the Introduction, ACYs are only a few of the many different phenolic compounds in berry fruits and other food plants, and the AA of a food or food extract will thus depend on the presence of these other phenolic compounds and their relative AA. Siriwoharn et al. (2004) reported mean total ACY concentrations of 131-256 $\mathrm{mg} / 100 \mathrm{~g}$ fruit, and TPH concentrations of 682-1056 mg/100 g fruit among 11 blackberry cultivars. Procyanidins, ellagitannins, and flavonols were the major polyphenolic classes they quantified in the fruit, and their respective concentrations varied among the cultivars. In 12 thornless blackberry selections and cultivars, the two flavonols identified by HPLC were quercetin and kaempferol 
(Bilyk and Sapers, 1986). Thus, it is not entirely surprising that, in blackberry (and other berry fruits), the TPH might more closely correlate with AA than ACY content does.

Some reports suggest that ACYs possess biological activities that may confer health benefits to humans. In some cases, the activities have been attributed to the AA of the ACYs (Serraino et al., 2003; Youdim et al., 2000) and differences in AA among various ACYs have been noted in several studies (Deighton et al., 2002; Fukumoto and Mazza, 2000; Tsuda et al., 1994; Wang et al., 1997). If ACYs substantially vary in AA, it raises the possibility that particular combinations of ACYs might be more efficacious than others in providing the AA necessary for those biological activities or health benefits dependent on high AA. The results from this study indicate that the ACYs occurring in extracts of blackberry or hybridberry fruit do not explain the variation in AA in those extracts as well as TPH does, regardless of whether they are considered as main effects or in interactions with each other or with location and year effects. This, in turn, suggests that phenolic compounds other than ACYs have substantial importance in determining AA in the fruit extracts, and that it may be the simple phenolic content or perhaps the phenolic profile as a whole, exclusive or inclusive of ACYs, that dictates the relative efficacy of a particular berry fruit or berry fruit extract in providing AA or the biological activities or presumed health benefits linked to AA.

It is possible that some of the biological activities demonstrated by ACYs may occur through non-AA-related mechanisms. For example, some reports addressing vascular health and function (Andriambeloson et al., 1998; Martin et al., 2003) and the moderation of lung inflammation in response to injury (Rossi et al., 2003) suggest that ACYs possess anti-inflammatory activity, or might modulate calcium concentrations that can influence apoptosis (programmed cell death). If this is the case, then a particular ACY profile, or specific ACY concentration in a food or extract, might be considered a goal or "target" for breeding or processing. The cultivar $x$ environment interactions that we observed in total and individual ACYs indicate that, if a particular ACY profile in blackberries or hybridberries is sought through breeding, considerable effort might be required to identify genotypes that provide this consistently well in all environments.

The partitioning of cultivars based on ACY profile that we obtained by linear discriminant analysis did not relate well to the coancestries of the parents. In a factorial study of red raspberry, Connor et al. (2005b) found that ACY profiles of progeny were poorly predicted based on knowledge of the parents. They suggested this would lead to difficulties for a breeding program that aimed to produce cultivars with specific ACY profiles. Our results with blackberries and hybridberries, although less robust, suggest that the same might apply for these Rubus species as well. Nevertheless, the presence in the profile of an ACY characteristic of a particular species is useful in detecting or confirming interspecific origins in genotypes exhibiting blackberry characters, as suggested by Jennings and Carmichael (1980). The presence of cy-sop in 'Boysen', 'Logan', 'Ranui', and 'Tayberry' coincides with the known or presumed red raspberry in the ancestry of these cultivars. However, 'Black Butte', 'Kotata', 'Marion', 'Silvan', and 'Siskiyou' all have red raspberry in their ancestry and yet did not produce detectable amounts of cy-sop.

In summary, our study demonstrated significant cultivar variation and important cultivar $\times$ environment interactions for total and individual ACYs among the 16 blackberry and hybridberry genotypes examined. Cultivar $\times$ year and cultivar $\times$ location together contributed from $7 \%$ to $24 \%$ of the variation in individual
ACYs, and $39 \%$ of the variation in total ACY.Cultivar interactions were also significant for AA, contributing $55 \%$ of the total variation. Phenotypic correlation was highest $(r=0.63)$ between total $\mathrm{ACY}$ and AA; this is substantially lower than the correlation $(r=$ 0.97 ) between total phenolic content and AA previously reported among these cultivars.

\section{Literature Cited}

Andriambeloson, E., C. Magnier, G. Haan-Archipoff, A. Lobstein, R. Anton, A. Beretz, J.C. Stoclet, and R. Andriantsitohaina. 1998. Natural dietary polyphenolic compounds cause endothelium dependent vasorelaxation in rat thoracic aorta. J. Nutr. 128:2324-2333.

Barritt, B.H. and L.C. Torre. 1975. Inheritance of fruit anthocyanin pigments in red raspberry. HortScience 10:526-528.

Benzie, I.F.F. and J.J. Strain. 1996. The ferric reducing ability of plasma (FRAP) as a measure of "antioxidant power": The FRAP assay. Anal. Biochem. 239:70-76.

Bilyk, A. and G.M. Sapers. 1986. Varietal differences in the quercetin, kaempferol, and myricetin contents of highbush blueberry, cranberry, and thornless blackberry fruits. J. Agr. Food Chem. 34:585-588.

Block, G., B. Patterson, and A. Subar. 1992. Fruit, vegetables, and cancer prevention: a review of the epidemiological evidence. Nutr. Cancer 18:1-29.

Cadet, J.L. and C. Brannock. 1998. Free radicals and the pathobiology of brain dopamine systems. Neurochemistry Intl. 23:117-131.

Clark, J.R., L. Howard, and S. Talcott. 2002. Antioxidant activity of blackberry genotypes. Acta Hort. 585:475-480.

Connor, A.M., C.E. Finn, and P.A. Alspach. 2005a. Genotypic and environmental variation in antioxidant activity and total phenolic content among blackberry and hybridberry cultivars. J. Amer. Soc. Hort. Sci. 130:527-533.

Connor, A.M., T.K. McGhie, M.J. Stephens, H.K. Hall, and P.A. Alspach. 2005b. Variation and heritability estimates of anthocyanins and their relationship to antioxidant activity in a red raspberry factorial mating design. J. Amer. Soc. Hort. Sci. 130:534-542.

Cooney, J.M., D.J. Jensen, and T.K. McGhie. 2004. LC-MS identification of anthocyanins in Boysenberry extract and in human urine following dosing. J. Sci. Food Agr. 84:237-245.

Deighton, N., R. Brennan, C. Finn, and H.V. Davies. 2000. Antioxidant properties of domesticated and wild Rubus species. J. Sci. Food Agr. 80:1307-1313.

Deighton, N., D. Stewart, H.V. Davies, P.T. Gardner, G.G. Duthie, W. Mullen, andA. Crozier. 2002. Soft fruit as sources of dietary antioxidants. Acta Hort. 585:459-465.

Esterbauer H., J. Gebicki, H. Puehl, and G. Jürgens. 1992. The role of lipid oxidation and antioxidants in modification of LDL. Free Radical Biol. Medicine 13:341-390.

Fukumoto,L.R. and G. Mazza. 2000. Assessing antioxidant and prooxidant activities of phenolic compounds. J. Agr. Food Chem. 48:3597-3604.

Harborne, J.B. and E. Hall. 1964. Plant polyphenols-XIII. The systematic distribution and origin of anthocyanins containing branched trisaccharides. Phytochemistry 3:453-463.

Hertog, M.G.L., E.J.M. Feskens, and D. Kromhout. 1997. Antioxidant flavonols and coronary heart disease risk. Lancet 349:699.

Jennings, D.L. and E. Carmichael. 1980. Anthocyanin variation in the genus Rubus. New Phytol. 84:505-513.

Knekt, P., J. Kumpulainen, R. Järvinen, H. Rissanen, M. Heliövaara, A. Reuanen, T. Hakulinen, and A. Aromaa. 2002. Flavonoid intake and risk of chronic diseases. Amer. J. Clinical Nutr. 76:560-568.

Leake, D.S. 1998. Effects of flavonoids on the oxidation of low-density lipoproteins, p. 253-276. In: C.A. Rice-Evans and L. Packer (eds.). Flavonoids in health and disease. Marcel Dekker, New York.

Markesbery, W.R. 1997. Oxidative stress hypothesis in Alzheimer's disease. Free Radical Biol. Medicine 23:134-147.

Martin, S., G. Giannone, R. Andriantsitohaina, and M.C. Martinez. 2003. Delphinidin, an active compound of red wine, inhibits endothelial cell apoptosis via nitric oxide pathway and regulation of calcium homeostasis. Brit. J. Pharmacology 139:1095-1102. 
Mazza, G. and E. Miniata. 1993. Small fruits, p. 85-130. In: Anthocyanins in fruits, vegetables, and grains. CRC Press, London.

Moyer, R.A., K.E. Hummer, C.E. Finn, B. Frei, and R.E. Wrolstad. 2002. Anthocyanins, phenolics, and antioxidant capacity in diverse small fruits: Vaccinium, Rubus, and Ribes. J. Agr. Food Chem. 50:519-525.

Mullen, W., J. McGinn, M.E.J. Lean, M.R. MacLean, P. Gardner, G.G. Duthie, T. Yokota, and A. Crozier. 2002. Ellagitannins, flavonoids, and other phenolics in red raspberries and their contribution to antioxidant capacity and vasorelaxation properties. J. Agr. Food Chem. 50:5191-5196.

Praticò, D. 2002. Alzheimer's disease and oxygen radicals: New insights. Biochem. Pharmacology 63:563-567.

Rossi, A., I. Serraino, P. Dugo, R. Di Paola, L. Mondello, T. Genovese, D. Morabito, G. Dugo, L. Sautebin, A.P. Caputi, and S. Cuzzocrea. 2003. Protective effects of anthocyanins from blackberry in a rat model of acute lung inflammation. Free Radical Res. 37:891-900.

Sapers, G.M., K.B. Hicks, A.M. Burgher, D.L. Hargrave, S.M. Sondey, and A. Bilyk. 1986. Anthocyanin patterns in ripening thornless blackberries. J. Amer. Soc. Hort. Sci. 111:945-950.

Serraino, I., L. Dugo, P. Dugo, L. Mondello, E. Mazzon, G. Dugo, A.P. Caputi, and S. Cuzzocrea. 2003. Protective effects of cyanidin3-O-glucoside from blackberry extract against peroxynitrite-induced endothelial dysfunction and vascular failure. Life Sci. 73:10971114.

Singleton, V.L., R. Orthofer, and R.M. Lamuela-Raventos. 1999.
Analysis of total phenols and other oxidation substrates and antioxidants by means of Folin-Ciocalteu reagent. Methods in Enzymology 299:152-178.

Siriwoharn, T., R.E. Wrolstad, C.E. Finn, and C.B. Pereira. 2004. Influence of cultivar, maturity, and sampling on blackberry (Rubus L. hybrids) anthocyanins, polyphenolics, and antioxidant properties. J. Agr. Food Chem. 52:8021-8030.

Steinmetz, K.A. and J.D. Potter. 1996. Vegetables, fruit and cancer prevention: A review. J. Amer. Dietetic Assn. 96:1027-1039.

Stintzing, F.C., A.S. Stintzing, R. Carle, B. Frei, and R.E. Wrolstad. 2002. Color and antioxidant properties of cyanidin-based anthocyanin pigments. J. Agr. Food Chem. 50:6172-6181.

Torre, L.C. and B.H. Barritt. 1977. Quantitative evaluation of Rubus fruit anthocyanin pigments. J. Food Sci. 42:488-490.

Tsuda, T., M. Watanabe, K. Ohshima, S. Norinobu, S-W. Choi, S. Kawakishi, and T. Osawa. 1994. Antioxidative activity of the anthocyanin pigments cyanidin $3-O-\beta-\mathrm{D}$-glucoside and cyanidin. $\mathrm{J}$. Agr. Food Chem. 42:2407-2410.

Venables, W.N. and B.D. Ripley. 1994. Linear statistical models: Automated model selection, p. 175-177. In: Modern applied statistics with S-Plus. Springer-Verlag, New York.

Wang, H., G. Cao, and R.L. Prior. 1997. Oxygen radical absorbance capacity of anthocyanins. J. Agr. Food Chem. 45:304-309.

Youdim, K.A., A. Martin, and J.A. Joseph. 2000. Incorporation of the elderberry anthocyanins by endothelial cells increases protection against oxidative stress. Free Radical Biol. Medicine 29:51-60. 